\title{
LOCALLY TAME CURVES AND SURFACES IN THREE-DIMENSIONAL MANIFOLDS ${ }^{1}$
}

\author{
O. G. HARROLD, JR.
}

1. Introduction. The program to be described here is concerned primarily with an imbedding problem in the topology of 3-manifolds. A preliminary remark or two will relate this problem with those of a more general nature.

The compact 1-manifold without boundary, i.e. the simple closed curve, has long been characterized both as a subset of the plane and as an abstract space. The Jordan curve theorem and its converse, due to Schoenflies, accomplish the former. The latter is accomplished, for instance, by the theorem of Wilder [29] ${ }^{2}$ that among the locally compact, locally connected continua, the simple closed curve is distinguished by the fact that for any pair of points $A$ and $B$, the continuum is a union of two irreducibly connected sets from $A$ to $B$ having in common only these points.

The compact orientable 2-manifolds without boundary likewise admit characterizations both as subsets of three-space, say, and as abstract spaces. The first step in the direction of the former was taken by Brouwer around 1912 and completed by Wilder in 1930 . For citations to the rather extensive literature relating to the latter we refer to van Kampen's article [21]. Specifically, however, it may be pointed out that among the Peano spaces, the topological 2-sphere is characterized elegantly as the set satisfying the Jordan curve theorem nonvacuously. Zippin established the result in this form and gave an analogous characterization of the closed 2-cell [31]. Had the first characterization of the 2-sphere as an abstract space followed its characterization as a subset of three-space, one would be tempted to feel that perhaps a characterization of the 3-cell or 3-sphere along the lines of Zippin's work for dimension 2 would await the characterization of the 3-cell, say, as a subset of three-space. There are two characterizations of the 3-sphere or 3-cell as abstract spaces published to date that we are aware of. One is due to Bing [5], the other due to Woodard [30]. Bing has used his characterization in the solution of several problems.

An address delivered before the Lexington Meeting of the Society on December 1, 1956, by invitation of the Committee to Select Hour Speakers for Southeastern Sectional meetings; received by the editors December 11, 1956.

1 This paper was supported in part by the National Science Foundation, G-2793.

2 Numbers in brackets refer to the bibliography at the end of the paper. 
2. The Schoenflies extension problem. It will be recalled that Schoenflies not only contributed a converse to the Jordan curve theorem in the plane but also gave a proof of the theorem itself. The basic idea used in the proof of the Jordan theorem was the ultimate in simplicity. If a homeomorphism $h$ is given from a standard circle to a curve $J$ in the same plane $\pi$ in such a way as to preserve orientation, then there is a homeomorphism of $\pi$ onto itself, call it $H$, which is an extension of $h$. Since a standard circle separates the plane and is the common boundary of each of its residual domains, the same properties are established for $J$ via the homeomorphism $H$. The problem, then, reduces to showing that $h$ has an extension $H$. The solution is referred to as the Schoenflies extension theorem.

In his thesis Antoine [4] considered many phases of the corresponding extension problem in three-space. Among the numerous examples in his paper one of the most remarkable is that of a compact, totally disconnected perfect set, i.e. a Cantor set, call it $P$, such that if $S$ is any topological 2-sphere in three-space whose interior and exterior both meet $P$, then $S$ meets $P$. Since every Cantor set in three-space is known to lie on some arc, this example implies there are arcs in three-space that cannot be thrown onto a linear interval by a homeomorphism acting on the whole space. (The explicit construction of such Cantor sets in $n$-space, $n \geqq 3$, and proofs of their properties have been carried out by Blankenship [8]. Thus "wild" arcs exist in every euclidean $n$-space, $E^{n}, n \geqq 3$. $)^{3}$

Generally, the problem Antoine sets for himself is this: Given a homeomorphism $h$ between two sets $A$ and $B$, does an extension of $h$ exist to some pair of neighborhoods $U$ and $V$ of $A$ and $B$, respectively? An interesting situation is that in which $U$ and $V$ are all of 3-space. In this fortuitous circumstance $A$ and $B$ are equivalent under the group of homeomorphisms of three-space. In general, if the required neighborhoods $U$ and $V$ exist, it will not be possible to extend the homeomorphism to all of space. The case in which $A$ is the boundary of a plane triangle and $B$ is a polyhedral trefoil knot suggests that in some cases $h$ may be extended to neighborhoods $U$ and $V$ of $A$ and $B$, but neither $U$ nor $V$ may be taken to be all of 3-space. Considerations of the knot-group may be used to establish this latter fact. By means of the set $P$ referred to above, we see there are cases in which not only may we fail to take $U$ and $V$ as all of three-space but $U$ and $V$ fail to exist at all as neighborhoods of $A$ and $B$, respectively. In a sense

\footnotetext{
3 The symbol $E^{n}$ stands for euclidean $n$-space with a fixed rectangular coordinate system.
} 
one is not surprised about the failure of extension possibilities for polyhedral closed curves $A$ and $B$ due to global differences in the manner in which they are imbedded. However, the failure of the extension to exist for any neighborhoods $U$ and $V$ of the nonpolyhedral arc $A$ and interval $B$ showed a type of phenomenon entirely unexpected at that time. Antione also includes in his examples a topological 2-sphere $A$ such that any homeomorphism of $A$ onto a standard 2-sphere cannot be extended to all space. Thus the Schoenflies method of proof of the Jordan curve theorem does not admit generalization to higher dimensions.

3. The extension theorem for polyhedra. In $1924 \mathrm{~J}$. W. Alexander published three short notes in the Proceedings of the National Academy dealing with these matters $[1 ; 2 ; 3]$. In the second of these notes a "horned" sphere is constructed failing to have the extension property. In the third note further properties of one of Antoine's sets are described.

In the first note Alexander proves that if a polyhedron in 3-space is a topological 2-sphere then both the interior and the exterior (in a compactified space) have closures that are closed 3-cells. (As a corollary to the proof, one finds, almost immediately, that a polyhedral torus always has at least one unknotted complementary domain.) Progress on certain problems of 3-dimensional topology such as triangulation theorems, limited analogues of the Schoenflies theorem, all of recent date, depend at some stage on the result for polyhedral 2 -spheres. In the thirty-three years that have elapsed since this was attained no generalization of this mild sounding result to higher dimensions has been forthcoming. It might be noted in passing that Bing's characterization of 3-space among Peano spaces by means of sequences of partitionings does not use this result.

4. Homotopy in the complement and consequences. In their paper on Uniform local connectedness and contractibility, Eilenberg and Wilder [9] consider the consequences of postulating homotopy uniform local connectedness of the complement of a topological $n-1$ sphere in $n$-space. They show that each component of the complement is in this case simply connected. (Of course uniform local connectedness in the homology sense over a proper coefficient group is always present in the appropriate dimensions.) Thus the domains complementary to Alexander's horned sphere are not both uniformly locally connected in the homotopy sense and simple alterations of the construction can destroy the contractibility of either domain.

The above mentioned results gave some hope that suitable homo- 
topy properties of a domain complementary to a topological $n-1$ sphere might lead to an extension theorem. That this hope was futile was shown by the paper by Fox and Artin on Some wild cells and spheres in 3-dimensional space [10]. In this paper an example is given of a topological 2-sphere whose complement is an open 3-cell, the closure of the complement is not a closed 3-cell. This shows, rather pointedly, that the homotopy properties of the complement, at least "in the large," cannot be expected to yield an analogue of the Schoenflies extension theorem. The wildness of the examples in the Fox and Artin paper is established by destroying the usual homotopy properties of the complement either in the large, in the small, or both. To establish the quoted properties for their sets the authors bring into play the knot group of the set in question. Since all of the complements studied in that paper require an infinite set of generators, their techniques were essentially new in this field. (The first application of such group techniques to topology wherein the group requires an infinite set of generators and the presentation is given explicitly is due to Newman and Whitehead [26] as far as the author is aware.)

Let us recall the terminology introduced in [10].

Let $K$ be a set in a geometric complex $C$. Then $K$ is said to be tamely imbedded in $C$ if and only if there is a homeomorphism of $C$ onto itself throwing $K$ onto a polyhedron. In the contrary case $K$ is called wildly imbedded in $C$. The set $K$ is called locally tamely imbedded at $p$ provided there is a neighborhood $N$ of $p$ and a homeomorphism $h_{p}$ of the closure of $N(=\bar{N})$ onto a polyhedron in $C$ such that $h_{p}(\bar{N} \cap K)$ is a polyhedron. If $K$ is locally tamely imbedded at every point, call $K$ locally tame. Clearly, if $K$ is tamely imbedded in $C$, it is locally tamely imbedded. The converse proposition is established in [6] and [25] for $n=3$.

The aim of the present program, in essence, is to discover when a particular topological type is locally tamely imbedded.

In 1949 the author considered complements of cells in $n$-space having uniformly abelian local fundamental groups [15]. Roughly speaking, this property means that small paths determining a product in the sense of the path group commute on a set of small diameter and uniformly so.

The principal result states: Let $C$ be a closed, topological $i$-cell, $i=1,2, \cdots, n$ in the euclidean $n$-sphere, $S^{n}$, such that $S^{n} \backslash C$ has uniformly abelian local fundamental groups then $\pi_{1}\left(S^{n} \backslash C\right)$ is trivial. An inspection of the method of proof for $i=n-2$ is instructive. For $n=3$ a simple arc such that each point of the arc has neighborhoods satisfying the stipulated conditions is shown to have a simply connected 
complement. A glance at the proof, however, shows that the local condition may be suppressed for one end-point. Consequently, an arc has a simply connected complement provided that each point, save possibly one end-point, has a local basis consisting of spherical neighborhoods, each having a closure that meets the arc in a connected polygon. Example 1.2 of [10] shows the arc may be wild.

A set is called locally polyhedral at $p$ if there are arbitrarily small neighborhoods of $p$ whose closure meets the set in a finite polyhedron. The above result may then be re-stated: An arc that is locally polyhedral save for one end-point has a simply connected complement. It is natural to ask if "almost" locally polyhedral spheres have complementary domains that are simply connected.

Moise and the author [20] showed that if a topological 2-sphere is locally polyhedral at all except possibly 3 points, at least one complementary domain is simply connected. If at most two points are exceptional, either both domains are simply connected or one domain has a closure that is a closed 3-cell. If only one point is exceptional, both domains are simply connected and at least one of them has a closure that is a closed 3-cell. The 3-cell, of course, need not be tame.

5. Covering an arc by almost polyhedral spheres. Inasmuch as a topological 2 -sphere that is locally polyhedral save for 1 or 2 points has complementary domains much like those of a polyhedral 2-sphere (even if it is wildly imbedded) it seemed that if an arc would possess coverings by topological spheres of arbitrarily small diameters that are almost polyhedral one might hope to enclose the arc by a strictly polyhedral sphere lying near the arc. That is, if the covering 2 -sphere associated with a point is required to be locally polyhedral save where it meets the arc and, if, at such common points, other such spheres exist enclosing these points of entry and exit along the arc, then the union of a finite collection of such spheres ought to have an unbounded complementary domain whose frontier is strictly polyhedral, and, if the spheres are properly related, this frontier should be a polyhedral 2-sphere. In view of the examples in [10] some restriction on the cardinal of the set wherein the almost polyhedral sphere meets the arc is necessary. The condition on the cardinality turns out to be the essential condition. By the approximation theorem of Bing [7], the almost polyhedral nature of the enclosing spheres may be proved in $E^{3}$.

6. Locally peripherally unknotted curves and surfaces. Let $C$ be a topological $k$-manifold with or without boundary in a euclidean space $E^{n}$. For $k=0$ we consider $C$ to be locally peripherally unknotted and 
proceed inductively: $C$ is said to be locally peripherally unknotted at $x$ provided that for each positive number $\epsilon$ there exists a closed $n$-cell $L$ of diameter less than $\epsilon$ whose interior contains $x$ and such that the common part of $C$ with the boundary of $L$ is a locally peripherally unknotted $k-1$ cell or $k-1$ sphere according as $x$ lies on the boundary of $C$ or not. This property is evidently invariant under homeomorphisms of $E^{n}$ on itself. It implies, for $k=1, n=3$, what was called property $\rho$ in [17]. A strictly polyhedral $C$ in $E^{3}$ has the property.

7. The enclosure property. If $C$ is a set in $E^{n}$ and $\epsilon$ positive it is convenient to say $C$ has the enclosure property provided that there is a polyhedral $n-1$ sphere in the $\epsilon$-neighborhood of $C$ whose interior contains $C$. In the compactified 3-space any set with an open 3-cell complement has this property and conversely for compact $C$. For arcs we may summarize: If $C$ is a tame arc, $C$ is locally peripherally unknotted; if $C$ is a locally peripherally unknotted arc, $C$ has the enclosure property. Neither implication may be reversed. (See Examples 1.4 and 1.2 of [10].)

8. Locally unknotted curves and surfaces. Let $M$ denote a closed $k$-cell and $\partial M$ its combinatorial boundary. Suppose $N$ is a closed $k-1$ cell contained in $M$ such that $(\partial M) \cap N$ is a $k-2$ sphere and under some parameterization of $M, N$ is a parameter $k-1$ cell in $M$. We say shortly that $N$ spans the boundary $\partial M$ of $M$. For $k=2$ every arc $N$ in $M$ whose intersection with the boundary is a pair of points, contains an arc that spans $\partial M$.

If $C$ is a topological $k$-manifold in $E^{n}$ with or without boundary, call $C$ locally unknotted at $x$ if and only if there is some $k+1$ cell $D$ such that $C \cap D$ is the closure of a neighborhood of $x$ in $C$ that lies on a spanning $k$-cell of $\partial D$. If $n=3, k=1$, this becomes what was called property $Q$ in the local form [18].

If $C$ is a simple closed curve in $E^{3}$ that bounds any 2-cell, it is locally unknotted at every point. Suppose $F$ is a 2-cell and $\partial F=C$. First we move $F$ slightly by a homeomorphism on $F$, keeping the points of $C$ fixed so that the image $F^{\prime}$ of $F$ is locally polyhedral at points ${ }^{4}$ of $F^{\prime} \backslash C$. Let $F^{\prime}$ become inflated to obtain a 3-cell $G$, again leaving the points of $C$ fixed, and $G$ locally polyhedral at points of $G \backslash C$. (The first step is justified by Bing's approximation theorem, the second step elementary on account of the polyhedral character of $F^{\prime}$ ). For any point $x$ of $C$ we may draw the desired 2-cell $D$ on $\partial G$.

Now, if it is also known that $C$ is locally peripherally unknotted,

4 The complement of $C$ in $F^{\prime}$ is denoted by $F^{\prime} \backslash C$. 
then by Theorem VII of [18], $C$ is tame and in fact bounds a tame 2cell. Thus $C$ is unknotted in the classical sense. Since locally tame arcs and simple closed curves are evidently locally unknotted and locally peripherally unknotted, we may say $a 1$-manifold $C$ is locally tame if and only if it is both locally unknotted and locally peripherally unknotted at every point.

In the first proof of the theorem to the effect that a curve that is locally unknotted and locally peripherally unknotted is locally tame, a 2-cell $D$ that is locally polyhedral modulo ${ }^{5}$ its boundary is postulated and used for a complicated auxiliary construction. The embedding of $D$ itself was not studied. In a later work (unpublished) the authors showed that $D$ is tame, and, as a corollary that $C=\partial D$ is tame [19]. (This result may also be gotten from Theorem VII of [18] and a theorem of Moise on "smoothing an annulus" [25].)

At this point we have attained a characterization of the 1-manifold and a fragmentary result for surfaces. The next sequence of steps seems clear. One might seek a more general result for surfaces or one might hope to tame more general 1-dimensional sets. Both steps will be necessary before attacking the general 2-dimensional complex.

In connection with the Example 1.4 referred to we have a sample of the difficulties to be expected when the imbedding of more general 1-dimensional sets than 1-manifolds is undertaken. This arc is a union of two tame arcs having in common only an end-point of each arc. At the end-point the union is locally knotted, hence the theorem about locally tame sets being tame cannot be applied. It is natural to expect that conditions specifying a tamely imbedded finite graph should be closely related to the conditions obtained for an arc or simple closed curve. Since an arc or simple closed curve is tame if and only if every sub-arc is tame, one might wonder if this condition would be necessary and sufficient for the tame imbedding of a finite graph. P. Doyle ${ }^{6}$ has recently exhibited an example of a triod such that every sub-arc is tame, the complement is an open 3-cell, but the triod itself is wildly imbedded. A form of local peripheral unknottedness (or property $\mathcal{P}$ ) applicable to 1-dimensional regular curves has been studied by C. Masaitis [23]. For finite dendrites he obtains an enclosure property analogous to that obtained for arcs. Since every dendrite, finite or not, has a homeomorphic image in the plane, it might be permissible to call a dendrite tame if some homeomorphism on space maps the dendrite into a subset of a plane.

${ }^{5}$ The set $D$ is locally polyhedral modulo $C$ if it is locally polyhedral at the points of $D \backslash C$.

${ }^{6}$ University of Tennessee thesis, 1957. 
Other known characterizations of tame curves will now be described before discussing surfaces explicitly.

9. Tame curve types have finite crookedness. If $P$ is a simple closed polygon, let $k(P)$ be the sum of suitably defined "external" angles measured along $P$ according to some selected orientation. For an arbitrary simple closed curve define $k(C)=1$.u.b. $k(P), P$ inscribed in $C$. If $\mathfrak{e}$ is the class of curves in $E^{3}$ whose members are equivalent to $C$ under some space isotopy, define $k(\mathfrak{e})=$ g.l.b. $k(C), C \in \mathbb{C}$. This number may be referred to as the total curvature of the class $\mathbb{e}$.

If $\bar{r}=\bar{r}(t)$ is a representation of the curve $C$, let $\mu(C, \bar{b})$ equal the number of maxima of the function $\bar{r} \cdot \bar{b}$ in a fundamental period $0 \leqq t \leqq 2 \pi$, where $\delta$ is a fixed unit vector. J. W. Milnor sets $\mu(C)$ $=\min _{b} \mu(C, b)$ and refers to $\mu(C)$ as the crookedness of $C$. For a class $\mathfrak{C}$, put $\mu(\mathfrak{e})=\min \mu(C), C \in \mathfrak{C}$. The fundamental relation between $k(\mathfrak{C})$ and $\mu(\mathfrak{C})$ is given by Milnor,

$$
2 \pi \mu(\mathfrak{C})=k(\mathfrak{e}) .
$$

The class of tame unknotted curves is then characterized by $k(\mathcal{C})$ $=2 \pi$, and, for a class of tame curves generally, $k(\mathbb{e})<+\infty$ [24]. Whether this device could be used to study the imbedding of other configurations seems not to have been investigated.

10. Tame curves and regular curve families. In $1933 \mathrm{H}$. Whitney defined regular curve families and later showed that a regular curve family filling an open set in $E^{2}$ or $E^{3}$ may be cross-sectioned $[27 ; 28]$. It is easily seen that this implies each trajectory of the family is locally tame. For if a point $p$ lies on a nondegenerate trajectory $J$, then nearby points $p^{\prime}, p^{\prime \prime}$ of the same trajectory such that $p^{\prime}<p<p^{\prime \prime}$ will determine cross sections $A^{\prime}, A^{\prime \prime}$ from which we can pick a pair of 1 -spheres $B^{\prime}, B^{\prime \prime}$, enclosing $p^{\prime}, p^{\prime \prime}$ on $A^{\prime}, A^{\prime \prime}$, respectively, that correspond under the parametrization. Then disks of $A^{\prime}, A^{\prime \prime}$ bounded by $B^{\prime}, B^{\prime \prime}$ and the ring swept out by following $B^{\prime}$ along until it falls on $B^{\prime \prime}$ give a topological 2-sphere enclosing $p$ that meets $J$ only at $p^{\prime}$ and $p^{\prime \prime}$. Evidently $J$ is locally peripherally unknotted at $p$. If we join $B^{\prime}$ to $p^{\prime}$ by an appropriate arc $\alpha$ in $A^{\prime}$ and consider the locus swept out by $\alpha$ as $p^{\prime}$ moves to $p^{\prime \prime}$, we see $J$ is locally unknotted ${ }^{7}$ at $p$. Hence $J$ is locally tame. It is obvious that a tame curve is (locally) a member of a regular curve family.

11. The enclosure properties for surfaces. In his thesis [12] H. C. Griffith generalized property $\odot$ to apply to $k$-cells in $n$-space. If $C$ is

7 To be exact, we see $J$ has local property $Q$ at $p$, then argue as in paragraph 8. 
a closed $k$-cell in $n$-space, Griffith requires that each parameter $k-1$ cell $T$ be enclosed in an arbitrary neighborhood of $T$ by a topological $n-1$ sphere that is locally polyhedral save where it meets $C$ and the common part of $C$ with the enclosing $n-1$ sphere must be a pair of spanning $k-1$ cells (or a single $k-1$ cell if $T$ lies on the boundary of $C$ ). The hypothesis that the intersection consists of spanning $k-1$ cells may be eliminated for $k=2, n=3$. The relation between this property and local peripheral unknottedness for $k=2, n=3$ is not clear at present. It is not difficult to see, however, that the above defined property $P$ does imply that the boundary of $C$ be tame. As we remarked earlier, the enclosure property implies $C$ has an open 3 -cell complement. But, whereas in the 1-dimensional case property $\odot$ implies the enclosure property, this has not been proved true for surfaces without further hypotheses.

Griffith defines a strong enclosure property as follows: a set $C$ has the strong enclosure property provided each pair $U, V$ of disjoint open subsets of $\partial C$ contain, respectively, the initial point of a pair of disjoint rays, each of which is otherwise disjoint from $C$, and which are such that every neighborhood of $C$ contains a polyhedral sphere enclosing $C$ and meeting each ray of the pair in a single point. There are examples to show that for a $k$-cell, $k \geqq 2$, the enclosure property does not imply the strong enclosure property.

12. The disk and uniform disk properties. Let $T$ be a spanning cell of the boundary $\partial C$ of $C$ or a subset of $\partial C$. In the former case let $M^{\prime}, N^{\prime}$ be the components of $C \backslash T$, in the latter case let $M^{\prime}$ be the component of $C \backslash T$ and $N^{\prime}$ null. Putting $M=M^{\prime} \cup T, N=N^{\prime} \cup T$, $T$ determines a triple $(T, M, N)$ of closed non-null sets. If $\sigma$ is the usual Hausdorff metric, put

$\rho\left(T_{1}, T_{2}\right)=\min \left\{\sigma\left(M_{1}, M_{2}\right)+\sigma\left(N_{1}, N_{2}\right), \sigma\left(M_{1}, N_{2}\right)+\sigma\left(N_{1}, M_{2}\right)\right\}$.

This is a metric over the collection of spanning cells of the boundary of $C$.

The $k$-cell $C$ is said to have the disk property provided that to each parameter $k-1$ cell $T$ of $C$ and positive $\epsilon$ there exists a set $D$ such that (i) $D$ is a disk; (ii) $\partial D \cap C=0$; (iii) $D \cap C$ is a parameter cell (not necessarily under the same parameterization that corresponds to $T$ ); (iv) If $C \backslash D$ has two components, then $D$ separates them in every sufficiently small neighborhood of $C$, and (v) $\rho(D \cap C, T)<\epsilon$.

If, as $\epsilon \rightarrow 0$, it is possible to choose $D$ so that the distance from $C$ to the boundary of $D$ does not approach $0, D$ is said to have the uniform disk property. 
If in the choice of $T$, we restrict ourselves to cells $T$ corresponding to a particular homeomorphism $h$ of $I^{k}$ onto $C^{k}, I=[0,1]$, the other conditions remaining unaltered, $C^{k}$ is said to have the disk property relative to $h$, or the uniform disk property relative to $h$, respectively.

Griffith proves these weaker forms of property $\beta$ and the uniform disk property together imply the enclosure property. Then, using the stated forms of property $\rho$ and the uniform disk property, the strong enclosure property follows. Finally, any two of the three properties imply the third. Recently, Griffith [13] has shown that among the 2-cells in 3-space having the disk property and strong enclosure property a tame 2-cell $C$ is characterized by the fact that if $D_{1}$ and $D_{2}$ are disks satisfying (i), (ii), (iii), and (iv) above and $T$ is a parameter 1-cell such that $T \cap D_{i}$ is a point, $i=1,2$, and $\epsilon>0$, then there exists a disk $D$ satisfying (i), (ii), (iii), (iv), and (v) for this $T$ and $\epsilon$ and such that $D \cap D_{i} \cap C$ is a point, for $i=1,2$.

13. Relations between the concepts of local unknottedness and local peripheral unknottedness. Examples 1.2 and 1.4 of [10] show that local unknottedness and local peripheral unknottedness are independent conditions for $k=1$ and $n=3$. For $k=2$ and $n=3$ local unknottedness implies local peripheral unknottedness as follows. If $p$ is in $C$, there is a 3-cell $D$ such that $C \cap D$ is (i) the closure of a neighborhood $M$ of $p$ in $C$, (ii) $\bar{M}$ lies on a spanning cell $K$ of $\partial D$. By definition of $D$ there is a homeomorphism $h$ of $D$ onto the locus $\sigma:\left\{(x, y, z) ; x^{2}+y^{2}+z^{2} \leqq 1\right\}$ and $h(K)$ may be taken to be a common part of $\sigma$ with the $x-y$ plane, since $K$ spans $\partial D$. If $\bar{M}=K$, we are through. If $\bar{M} \neq K$, then $\bar{M}$ is a closed 2-cell with $p$ on its boundary. The Schoenflies extension theorem may be applied to map $h(\bar{M})$ onto the locus $\sigma^{\prime}:\left\{(x, y, z) ; x^{2}+y^{2} \leqq 1, z=0, y \geqq 0\right\}$. This generates a homeomorphism of $\sigma$ on itself in an obvious manner so that the final image of $D$ is a sphere and the image of $\bar{M}$ is a semi-circular planar disk. Since $\sigma$ could have been taken as a standard simplex, this shows $C$ is actually locally tame, hence, as a corollary, locally peripherally unknotted.

The condition local peripheral unknottedness is weaker than local unknottedness for $k=2$ as may be seen by the following example, communicated by H. C. Griffith and R. L. Plunkett. Let $C$ be obtained from a planar rectangle by removing the interiors of a sequence of disjoint circles converging to the center of the rectangle. Fill in each open circular hole by a disk of diameter not more than twice the diameter of the hole which has the imbedding of the example 1.2 of [10] when that arc is replaced by a disk. The set $C$ so formed is a disk that is locally peripherally unknotted at the center. It is not 
locally tame at this point, hence locally knotted. The possibility remains that if a disk $C$ is locally peripherally unknotted at every point that $C$ would be locally unknotted at each point.

14. Extension problems in euclidean 4-space. The definition in $\S 6$ above shows that if $C$ is a locally peripherally unknotted manifold in $E^{n}$ and $E^{n}$ is regarded as a hyperplane of $E^{n+m}$, then $C$ is locally peripherally unknotted in $E^{n+m}$. Similarly, if $C$ is locally unknotted in $E^{n}$, it is automatically locally unknotted in $E^{n+m}$. The extent to which these properties may characterize tame curves and surfaces in $E^{n}, n>3$, is open.

It will be noticed that the definition of "tameness" for $n>3$ is less restrictive than for $n=3$ in the sense that a $k$-cell that is tame in $E^{4}$ may or may not be "flat," i.e. there may exist no semi-linear homeomorphism on $E^{4}$ carrying a polyhedral 2-cell into a subset of some $E^{2}$ in $E^{4}$. For instance, if $C$ is the join of a polyhedral knot in $E^{3}$ and a point $p$ of $E^{4} \backslash E^{3}$, then $C$ is polyhedral, hence tame, but not flat in the sense of Gugenheim [14]. It is easy to see that $C$ is locally peripherally unknotted in $E^{4}$. The decision as to whether $C$ is locally knotted at $p$ or not is resolved less easily. (It is evident that no "flat" 3-cell $D$ exists in $E^{4}$ of which $C$ is a spanning 2-cell for $\partial D$.)

The construction of Antoine may be used to show that there are 1 -cells in $E^{n}$ that are locally peripherally knotted. Presumably there might be a 1-cell in $E^{4}$ that lies on no 2-cell in $E^{4}$ and perhaps a 2-cell in $E^{4}$ that lies on no 3-cell. Fulfillment of both of these conditions would be necessary for a 1-cell in $E^{4}$ to be equivalent to a flat 1-cell. Thus, even a locally unknotted and locally peripherally unknotted arc in $E^{4}$ might fail to be equivalent to a segment because of the lack of existence of the appropriate 3-cell.

V. L. Klee has an extension theorem for subsets of linear normed spaces that may be used to show that every arc in $E^{8}$ is tame in $E^{4}$ (even "flat") [22, Theorem 3.3]. Hence every simple closed curve in $E^{3}$ is locally tame in $E^{4}$. If $J$ is a simple closed curve in $E^{3}$ it may be shown that it bounds a disk in $E^{4}$ that is locally polyhedral modulo $J$. It is not clear such a disk would be locally tame at the points of $J$.

The immediate prospect of obtaining extension theorems in 4-space analogous to those quoted above in paragraphs 8,12 seems remote until a suitable generalization or substitute is found for the Alexander theorem. Since a polyhedral 2-sphere may be knotted in $E^{4}$, it would appear a classification of the ways in which such a sphere may be imbedded in $E^{4}$ would be an essential step. R. H. Fox and J. W. Milnor have extended results of Gugenheim in this direction $[11 ; 14]$. 


\section{BIBLIOGRAPHY}

1. J. W. Alexander, On the sub-division of space by a polyhedron, Proc. Nat. Acad. Sci. U.S.A. vol. 10 (1924) pp. 6-8.

2. - An example of a simply connected surface bounding a region which is not simply connected, ibid. vol. 10 (1924) pp. 8-10. 10-12.

3. - Remarks on a point set constructed by Antoine, ibid. vol. 10 (1924) pp.

4. L. Antoine, Sur l'homeomorphie de deux figures et leurs voisinages, J. Math. Pures Appl. Series 8 vol. 4 (1921) pp. 221-325.

5. R. H. Bing, A characterization of 3-space by partitionings, Trans. Amer. Math. Soc. vol. 70 (1951) pp. 15-27.

6. - Locally tame sets are tame, Ann. of Math. vol. 59 (1954) pp. 145-158.

7. —_, Approximating surfaces by polyhedral ones, to appear.

8. W. A. Blankenship, Generalization of a construction of Antoine, Ann. of Math. vol. 53 (1951) pp. 276-297.

9. S. Eilenberg and R. L. Wilder, Uniform local connectedness and contractibility, Amer. J. Math. vol. 64, pp. 613-622.

10. R. Fox and E. Artin, Some wild cells and spheres in 3-dimensional space, Ann. of Math. vol. 49 (1948) pp. 979-990.

11. R. Fox and J. W. Milnor, Knotted 2-spheres in 4-dimensional space.

12. H. C. Griffith, The enclosing of cells in 3-space by simple closed surfaces, Trans. Amer. Math. Soc. vol. 81 (1956) pp. 25-48.

13. - A characterization of tame surfaces in 3-space.

14. V. K. A. M. Gugenheim, Piecewise linear isotopy and embedding of elements and spheres, I, Proc. London Math. Soc. Ser. 3 vol. 3, pp. 29-53; II. ibid. pp. 129-152.

15. O. G. Harrold, Jr., Euclidean domains with uniformly abelian local fundamental groups, Trans. Amer. Math. Soc. vol. 67 (1949) pp. 120-129.

16. - The enclosing of simple arcs and curves by polyhedra, Duke Math. J. vol. 21 (1954) pp. 615-622.

17. - Some consequences of the approximation theorem of Bing, Proc. Amer. Math. Soc. vol. 8 (1957) pp. 204-206.

18. O. G. Harrold, H. C. Griffith and E. E. Posey, A characterization of tame curves in 3-space, Trans. Amer. Math. Soc. vol. 79 (1955) pp. 12-35.

19. - The imbedding in three-space of cells which are locally polyhedral except at boundary points, Bull. Amer. Math. Soc. Abstract 61-1-121.

20. O. G. Harrold and E. E. Moise, Almost locally polyhedral spheres, Ann. of Math. vol. 57 (1953) pp. 575-578.

21. E. R. van Kampen, On some characterizations of 2-dimensional manifolds, Duke Math. J. no. 1 (1935) pp. 74-93.

22. V. L. Klee, Some topological properties of convex sets, Trans. Amer. Math. Soc. vol. 78 (1955) pp. 30-45.

23. C. Masaitis, Complement of a finite dendrite in the compactified 3-space, Bull. Amer. Math. Soc. Abstract 62-1-118.

24. J. W. Milnor, On the total curvature of knots, Ann. of Math. vol. 52 (1950) pp. 248-257.

25. E. E. Moise, Affine structures in 3-manifolds, VIII, Ann. of Math. vol. 59 (1954) pp. 159-170.

26. M. H. A. Newman and J. H. C. Whitehead, The group of a certain linkage, Quart. J. Math. Oxford Ser. vol. 8 (1937) p. 14. 
27. H. Whitney, Regular families of curves, Ann. of Math. vol. 34 (1933) pp. 244270.

28. - Cross-sections of curves in 3-space, Duke Math. J. vol. 4 (1939) pp. 222-226.

29. R. L. Wilder, Concerning simple continuous curves and related point sets, Amer. J. Math. vol. 53 (1931) pp. 39-55.

30. D. W. Woodard, The characterization of the closed n-cell, Trans. Amer. Math. Soc. vol. 42 (1937) pp. 396-415.

31. L. Zippin, A characterization of the closed 2-cell, Amer. J. Math. vol. 55 (1933) pp. 207-217.

University of Tennessee 